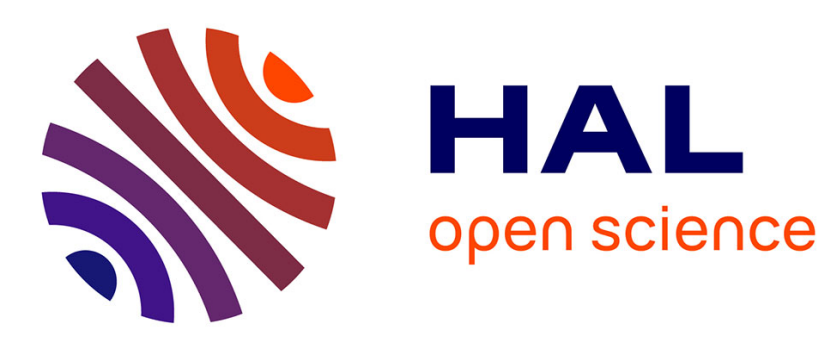

\title{
Cinétique du transport de matière dans un tube ouvert
} Joël Chevrier

\section{To cite this version:}

Joël Chevrier. Cinétique du transport de matière dans un tube ouvert. Revue de Physique Appliquée, 1977, 12 (6), pp.931-936. 10.1051/rphysap:01977001206093100 . jpa-00244263

\section{HAL Id: jpa-00244263 https://hal.science/jpa-00244263}

Submitted on 1 Jan 1977

HAL is a multi-disciplinary open access archive for the deposit and dissemination of scientific research documents, whether they are published or not. The documents may come from teaching and research institutions in France or abroad, or from public or private research centers.
L'archive ouverte pluridisciplinaire HAL, est destinée au dépôt et à la diffusion de documents scientifiques de niveau recherche, publiés ou non, émanant des établissements d'enseignement et de recherche français ou étrangers, des laboratoires publics ou privés. 
Classification

Physics Abstracts

9.210

\title{
CINÉTIQUE DU TRANSPORT DE MATIẺRE DANS UN TUBE OUVERT
}

\author{
J. CHEVRIER \\ Centre d'Etudes d'Electronique des Solides \\ Université des Sciences et Techniques du Languedoc, Place Eugène Bataillon, 34060 Montpellier, France
}

(Reçu le 6 janvier 1977, accepté le $1^{\mathrm{er}}$ mars 1977)

\begin{abstract}
Résumé. - La cinétique du transport chimique en phase vapeur, dans un tube ouvert, a été étudié pour le système $\mathrm{ZnSe}-\mathrm{HCl}-\mathrm{H}_{2}$. Un modèle théorique, basé sur les lois générales de diffusionconvection a été développé pour pouvoir décrire le transfert de matière, depuis une zone chaude vers une zone froide. Un très bon accord avec l'expérience a été obtenu, malgré les hypothèses simples envisagées.
\end{abstract}

\begin{abstract}
The vapour phase chemical kinetics for the $\mathrm{ZnSe}-\mathrm{HCl}-\mathrm{H}_{2}$ system were studied in a horizontal open tube furnace. A theoretical model, based upon the general diffusion and convection laws has been developed to describe the matter transport from the hot source in the direction of a cold substrate. The experimental data were in good agreement with the theoretical predictions in spite of the simple hypotheses applied.
\end{abstract}

1. Introduction. - La cinétique de transfert de matière en phase vapeur, d'une source chaude vers une zone froide, a été abordé dans de nombreux articles, mais essentiellement pour le transport en tube fermé ([1] à [9]). Nous avons développé ici la cinétique du transport de matière en tube ouvert, beaucoup moins étudiée que la précédente ([10] à [12]) et ayant donné lieu à peu de comparaisons théorieexpérience. L'étude de ce transfert de matière au sein d'une phase vapeur est importante car elle permet ensuite de mieux aborder et d'approfondir la cinétique de dépôt sur un substrat, généralement positionné en zone froide $\left(T_{\mathrm{D}}\right)$.

Nous intéressant à la croissance de composés II-VI, nous avons développé un modèle théorique, basé sur les lois générales de diffusion-convection, et concernant le transport, dans un tube ouvert, d'une source solide $A(\mathrm{~s})$ de séléniure de zinc (ZnSe), maintenue à une température constante $T_{1}$, balayée par un flux gazeux réactif $\mathrm{H}_{2}+\mathrm{HCl}$. Le four impose en fait deux paliers de températures (à $T_{1}$ et $T_{\mathrm{D}}$ ) distants environ de $20 \mathrm{~cm}$. Le montage utilisé a été décrit dans un précédent article [13].

Nous avons pu décrire avec une très bonne approximation les phénomènes de transfert de $\mathrm{ZnSe}$ au sein de la phase vapeur, au cours de l'écoulement gazeux imposé.

2. Cinétique de transport. - 2.1 ÉQUATIONS GÉNÉRALES. - La plupart des études théoriques effectuées sur la cinétique de transport d'une espèce en phase vapeur reposent sur l'équation générale

$$
\frac{\partial c_{\mathrm{A}}}{\partial t}-\mathbf{\nabla} \mathbf{J}_{\mathrm{A}}+R_{\mathrm{A}}
$$

qui exprime la variation de la concentration molaire en espèce « $\mathrm{A} », c_{\mathrm{A}}$, en un point du système gazeux. $R_{\mathrm{A}}$ est le taux de production de $\mathrm{A}$, par unité de volume, par une réaction chimique éventuelle en phase vapeur. Nous admettrons qu'aux températures utilisées (de $600^{\circ} \mathrm{C}$ à $900^{\circ} \mathrm{C}$ ), les équilibres s'établissent instantanément en phase vapeur et que $R_{\mathrm{A}}=0$. Par contre, la vitesse des réactions chimiques entre la phase vapeur et le milieu condensé pourra jouer un rôle important sur la cinétique de transfert de la source solide au milieu gazeux; la production des espèces atomiques $\mathrm{Zn}$ et Se par ce type de réaction sera prise en compte dans les conditions aux limites du volume gazeux.

Dans l'équation (1), $J_{\mathrm{A}}$ est le flux molaire de $\mathrm{A}$, tel que

$$
\mathbf{J}_{\mathrm{A}}=-\mathfrak{D}_{\mathrm{A}} \nabla c_{\mathrm{A}}+c_{\mathrm{A}} \mathbf{v}
$$

$\mathfrak{D}_{\mathrm{A}}$ est le coefficient de diffusion de A dans le milieu gazeux $v$ est la vitesse massique moyenne définie par rapport à un repère fixe.

Le premier terme de (2) correspond au flux de diffusion donné par la loi de Fick; le second, au flux de convection de A résultant du mouviment 
d'ensemble du fluide. En se limitant au seul régime dit permanent, pour lequel on a, en tout point du mélange gazeux

$$
\frac{\partial c_{\mathrm{A}}}{\partial t}=0
$$

l'équation (1) devient

$$
D_{\mathrm{A}} \nabla^{2} c_{\mathrm{A}}-\nabla \cdot c_{\mathrm{A}} \mathbf{v}=0
$$

soit

$$
\mathfrak{D}_{\mathrm{A}} \nabla^{2} c_{\mathrm{A}}-c_{\mathrm{A}} \nabla \cdot \mathrm{v}-\mathbf{v} \cdot \nabla c_{\mathrm{A}}=0
$$

et, par conséquent, dans le cas général, il est indispensable de connaître l'évolution dans l'espace du champ de vitesse $\mathbf{v}$; celle-ci est décrite, pour un écoulement laminaire, à masse volumique $(\rho)$ et viscosité $(\mu)$ constantes, par un système de trois équations du type

$$
\rho \frac{\partial v_{x}}{\partial t}+\rho \mathbf{v} \cdot \nabla \mathbf{v}_{x}-\mu \nabla^{2} v_{x}
$$

pour la composante $v_{x}$. En fait, dans le cas d'une fluide incompressible $(\boldsymbol{\nabla} \cdot \mathbf{v}=0)$; les expressions (5) et (6) s'écrivent plus simplement

$$
\begin{array}{r}
\mathscr{D}_{\mathrm{A}} \nabla^{2} c_{\mathrm{A}}-\mathbf{v} \cdot \nabla c_{\mathrm{A}}=0 \\
v \nabla^{2} v_{x}-\mathbf{v} \cdot \nabla \mathbf{v}_{x}=0
\end{array}
$$

où $v$ est la viscosité cinématique du gaz vecteur $(\mu / \rho)$.

On remarque l'identité formelle de ces deux équations; pour les résoudre, il faut se fixer un modèle géométrique et des conditions aux limites correspondant au problème étudié.

2.2 Particularisation du PROBlème. 2.2.1 Approximation dé base. - Le modèle géométrique retenu est décrit sur la figure 1. La source présente une longueur « $l$ » dans la direction du courant gazeux, et une dimension " $a$ » dans la direction perpendiculaire, soit une surface réactive $A=a l$.

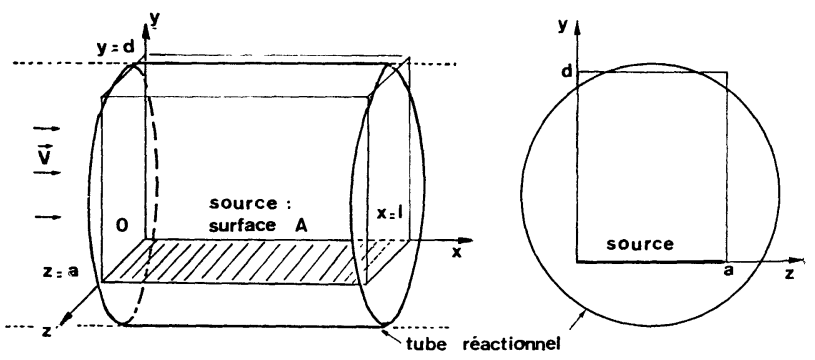

FIG. 1. - Schéma de la zone source et modèle géométrique retenu.

[Geometrical model considered in our study.]

Comme la géométrie du système n'est pas simple, nous avons choisi de l'approcher par un modèle à deux dimensions: La paroi du tube est figurée par une surface plane fictive, parallèle au plan de la source et située à une distance moyenne « $d$ » (direction $y$ ).

Nous avons alors fait abstraction du flux de matière pouvant quitter la zone source par simple effet de diffusion entre cette zone et les deux zones froides amont et aval, du fait des gradients thermiques existant. On se place donc en régime isotherme. Nous verrons cependant que le calcul des simples flux de diffusion et de rétrodiffusion, à faible vitesse, est possible et que ces derniers peuvent être simplement ajoutés au flux découlant de la solution du problème isotherme. Ce dernier est régi par les équations de conservations

$$
\begin{gathered}
\mathcal{D}\left(\frac{\partial^{2} c}{\partial x^{2}}+\frac{\partial^{2} c}{\partial y^{2}}\right)-v_{x} \frac{\partial c}{\partial x}-v_{y} \frac{\partial c}{\partial y}=0 \\
v\left(\frac{\partial^{2} v_{x}}{\partial x^{2}}+\frac{\partial^{2} v_{x}}{\partial y^{2}}\right)-v_{x} \frac{\partial v_{x}}{\partial x}-v_{y} \frac{\partial v_{x}}{\partial x}=0 .
\end{gathered}
$$

On a supprimé l'indice « $\mathrm{A}$ » pour plus de clarté et on néglige l'équation du mouvement en $v_{y}$ car cette composante ne doit pas jouer un rôle très important sur le mouvement d'ensemble du fluide.

2.2.2. Approximation du champ de vitesses uniforme. - Nous avons estimé que, compte tenu des faibles dimensions de la source par rapport au tube, le champ de vitesses ne pouvait être considérablement perturbé, surtout pour les vitesses linéaires mises en jeu ici $\left(v_{0}\right.$, définie à la température ambiante $T_{0}$, variant de 0,1 à $2 \mathrm{~cm} \cdot \mathrm{s}^{-1}$ ) ; aussi, avons nous considéré que ce champ était uniforme et parallèle à l'axe du tube. L'équation (10) n'est alors plus nécessaire et l'équation (9) se réduit à :

$$
\mathcal{D}\left(\frac{\partial^{2} c}{\partial x^{2}}+\frac{\partial^{2} c}{\partial y^{2}}\right)-v \frac{\partial c}{\partial x}=0
$$

où $v$ (pour $v_{x}$ ) devient la vitesse linéaire définie à la température $T$.

2.2.3 Conditions aux limites. - Les conditions aux limites imposées à la concentration $c$ sont :

a) Un flux de matière nul à la frontière supérieure en $y=d$, soit conformément à l'équation (2) :

$$
\left.J_{y}\right|_{y=d}=-\left.\mathfrak{D} \frac{\partial c}{\partial y}\right|_{y=d}=0 .
$$

b) Un flux de matière proportionnel à l'écart entre la concentration réelle et la concentration d'équilibre, à la surface de la source (en $y=0)$, soit

$$
\left.J_{y}\right|_{y=0}=k\left(c_{1}-c(x, 0)\right)
$$

$c_{1}$ est la concentration molaire en espèce gazeuse $\mathrm{ZnSe}$ dans la phase vapeur lorsque celle-ci est en équilibre avec le solide ; $k$ est le coefficient de trans- 
fert local de matière à l'interface solide-gaz; il dépend essentiellement de la vitesse de la réaction chimique

$$
\mathrm{ZnSe}(\mathrm{s})+\mathrm{HCl}(\mathrm{g}) \rightleftharpoons \mathrm{ZnCl}_{2}(\mathrm{~g})+\mathrm{SeH}_{2} \text {. }
$$

c) En régime isotherme, aucune condition ne peut être imposée dans le plan $x=l$. Il faut simplement que si $l$ tend vers l'infini, le profil de concentration $c(l, y)$ tende vers la concentration d'équilibre $c_{1}$.

d) La principale difficulté du problème réside en la définition d'un profil de composition dans la phase vapeur, à l'entrée de la zone source dans le plan $y 0 z$. Il faut cependant que :

- en régime permanent, à vitesse d'entraînement nulle, la composition de la phase vapeur dans ce plan soit homogène et égale à celle d'équilibre $c_{1}$;

- à vitesse d'entraînement non nulle, $c(0, y)$ s'écarte rapidement de $c_{1}$ quand la vitesse augmente ;

- cet entraînement doit être d'autant plus rapide que le coefficient de diffusion $\mathcal{D}$ est grand et que la distance $d$ est faible.

Nous avons pu résoudre notre problème en posant

que, dans le plan $y 0 z$, le flux moyen de matière restait constamment nul, quelles que soient les conditions de températures et de vitesses. Il en a résulté une concentration $c(0, y)$ répondant bien aux critères énoncés ci-dessus, mais une expression du flux de matière valable essentiellement à moyennes et grandes vitesses (qui sont les gammes utilisées d'une façon générale).

2.3 RÉSOLUTION DE L'ÉQUATION PRINCIPALE. - Avec les conditions aux limites imposées, la méthode de séparation des variables peut s'appliquer pour résoudre l'équation (11) en introduisant la fonction

$$
c^{\prime}(x, y)=c_{1}-c(x, y)
$$

et en posant

$$
c^{\prime}(x, y)=X(x) . Y(y)
$$

$c^{\prime}(x, y)$ doit vérifier l'équation générale

$$
\mathfrak{D}\left(\frac{\partial^{2} c^{\prime}}{\partial x^{2}}+\frac{\partial^{2} c^{\prime}}{\partial y^{2}}\right)-v \frac{\partial c^{\prime}}{\partial x}=0
$$

La solution générale de cette équation est de la forme

$$
c^{\prime}(x, y)=\sum_{m}\left[A_{m} \cos \lambda_{m} y+B_{m} \sin \lambda_{m} y\right] \exp \left(-\tau_{m} x\right)
$$

avec

$$
\tau_{m}=\frac{v}{2 \mathfrak{D}}\left[\left(1+\frac{4 \lambda_{m} 2 \mathfrak{D}^{2}}{v^{2}}\right)^{1 / 2}-1\right](>0)
$$

Compte tenu des équations (12), (13) et (14), on établit

$$
\begin{aligned}
c(x, y)=c_{1}\left[1-\frac{2}{d} \sum_{m} \exp \left(-\tau_{m} x\right)\right. & \left.\frac{\gamma_{m}}{\gamma_{m}+\frac{1}{h d}} \frac{\sin \lambda_{m} d}{\lambda_{m}} \cos \lambda_{m}(y-d)\right]+ \\
& +\frac{2}{d} \sum_{m} \exp \left(-\tau_{m} x\right) \cdot \frac{\gamma_{m}}{\gamma_{m}+\frac{1}{h d}} \cos \lambda_{m}(y-d) \int_{0}^{d} c\left(o, y^{\prime}\right) \cos \lambda_{m}\left(y^{\prime}-d\right) \mathrm{d} y^{\prime}
\end{aligned}
$$

avec

$$
\gamma_{m}=1+\frac{\lambda_{m}^{2}}{h^{2}} \quad \text { et } \quad h=\frac{k}{\mathfrak{D}}
$$

et $\lambda_{m}$ solution de

$$
\left(\lambda_{m} d\right) \operatorname{tg}\left(\lambda_{m} d\right)=k d / D
$$

Les racines de cette équation sont tabulées [14] [15].

Dans le cadre des approximations envisagées, cette expression est très générale, mais elle est fonction du coefficient local de transfert « $k »$, pratiquement inconnu pour le système étudié ici, et qui doit dépendre d'une certaine façon de la température et de la vitesse. Aussi avons nous adopté, pour $k d / \mathfrak{D}$, une valeur infinie, ce qui correspond au cas où l'équilibre thermodynamique est constamment réalisé en tout point de l'interface solide-gaz ; alors,

$$
\lambda_{m}=(2 m+1) \frac{\pi}{2 d}
$$


avec $m$ entier, positif ou nul soit

$$
\sin \left(\lambda_{m} d\right)=(-1)^{m} \quad \text { et } \quad \gamma_{m}=1
$$

Il en résulte une expression de la concentration plus simple

$$
c(x, y)=c_{1}\left\{1-2 \sum_{m=0} \exp \left(-\tau_{m} x\right) \cos \lambda_{m}(y-d)\left[\frac{(-1)^{m}}{\lambda_{m} d}-\frac{J_{m}}{2}\right]\right\}
$$

où $J_{m}$ vaut

$$
\frac{2}{d} \int c(0, y) \cos \lambda_{m}(y-d) \mathrm{d} y .
$$

Alors, l'imposition d'un flux moyen nul à l'entrée, défini préalablement par

$$
G_{0}=a \int_{0}^{d}-\left.\mathfrak{D} \frac{\partial c}{\partial x}\right|_{x=0}+v c(0, y) \mathrm{d} y
$$

entraîne la connaissance des « $J_{m}$ ", donc de la distribution de matière dans le volume au-dessus de la source.

Nous trouvons

$$
J_{m}=2 c_{1} \frac{(-1)^{m}}{\lambda_{m} d} \frac{\tau_{m} \mathfrak{D} / v}{1+\tau_{m} \mathfrak{D} / v}
$$

soit

$$
c(x, y)=c_{1}\left\{1-2 \sum_{m} \frac{(-1)^{m}}{\lambda_{m} d} \exp \left(-\tau_{m} x\right) \cos \lambda_{m}(y-d) \frac{1}{1+\tau_{m} \mathfrak{D} / v}\right\}
$$

Cette expression redonne bien, lorsque $v$ tend vers zéro, $c(x, y)=c_{1}$, ce qui signifie qu'en l'absence d'entrainement, en régime permanent, la composition de la phase vapeur est homogène et égale à celle d'équilibre. Remarquons encore que $c(0, y)$ satisfait bien aux criètres que nous nous étions imposés.

3. Flux de matière en zone source. - Le flux $G_{0}$, en $x=0$, étant supposé constamment nul, le flux d'interface $G_{i}$, dans la direction $0 y$, sera nécessairement équivalent au flux moyen $G_{l}$, en $x=l$, dans la direction $0 x$. A partir de l'expression de $c(x, y)$ établie et de la condition

$$
G_{\mathrm{i}}=a \int_{0}^{l}-\left.\mathfrak{D} \frac{\partial c}{\partial x}\right|_{y=0} \mathrm{~d} x
$$

nous obtenons facilement

$$
G_{\mathrm{i}}=2(a d) v c_{1} \sum_{m} \frac{1-\exp \left(-\tau_{m} l\right)}{\left(\lambda_{m} d\right)^{2}}
$$

Ce flux est nul à vitesse nulle, et tend vers une valeur positive, non nulle, lorsque cette vitesse tend vers l'infini ; cette valeur limite, traduisant physiquement une sous-saturation limite de la phase vapeur, vaut :

$$
\frac{2(a d) D l}{d^{2}}
$$

$(a d) v c_{1}$ correspond au régime de saturation qui sera retrouvé expérimentalement pour de faibles valeurs de $v$ ou de grandes longueurs de source.
4. Détermination des données de base. - L'application de l'expression établie nécessite la connaissance de la concentration d'équilibre $c_{1}$, donc de la pression d'équilibre $p_{1}$ d'une espèce ficive $\mathrm{ZnSe}(\mathrm{g})$, en phase vapeur, ainsi que celle du coefficient de diffusion de cette espèce (ou d'une espèce équivalente) dans le milieu gazeux.

4.1 Pressions Partielles D'ÉQuilibre. - Le modèle thermodynamique correspondant au système étudié ici comprend trojs équilibres chimiques principaux reliant six espèces gazeuses et une solide

$$
\begin{aligned}
\mathrm{ZnSe}(\mathrm{s})+\mathrm{HCl}(\mathrm{g}) & \rightleftharpoons \mathrm{ZnCl}_{2}(\mathrm{~g})+1 / 2 \mathrm{Se}_{2}(\mathrm{~g}) \\
2 \mathrm{ZnSe}(\mathrm{s}) & \rightleftharpoons 2 \mathrm{Zn}(\mathrm{g})+\mathrm{Se}_{2}(\mathrm{~g}) \\
\mathrm{H}_{2}(\mathrm{~g})+1 / 2 \mathrm{Se}_{2}(\mathrm{~g}) & \rightleftharpoons \mathrm{SeH}_{2}(\mathrm{~g}) .
\end{aligned}
$$

Les six pressions partielles inconnues peuvent être calculées numériquement en résolvant l'ensemble des six équations suivantes

$$
\begin{gathered}
P_{0}=\sum_{i} p_{i} \quad i=1,2, \ldots, 6 \quad P_{0}=\begin{array}{c}
1 \mathrm{~atm} . \\
\text { (tube ouvert) }
\end{array} \\
K_{m}=\prod_{i} p_{i}^{q i m} \quad m=1,2,3 . \\
(\mathrm{Cl} / \mathrm{H})=\sum_{i} v_{i, \mathrm{Cl}} p_{i} \sum_{i} v_{i, \mathrm{H}} p_{i} \\
(\mathrm{Zn} / \mathrm{Se})=\sum_{i} v_{i, \mathrm{Zn}} p_{i} \sum_{i} v_{i, \mathrm{Se}} p_{i} \quad \text { avec } \quad \mathrm{Zn} / \mathrm{Se}=1 .
\end{gathered}
$$

$v_{\mathrm{i}}, \mathrm{Zn}_{\mathrm{n}}, v_{\mathrm{i}, \mathrm{Se}}, v_{\mathrm{i}, \mathrm{Cl}}, v_{\mathrm{i}, \mathrm{H}}$ sont les coefficients stæchiométriques des atomes $\mathrm{Zn}$, Se, Se et $\mathrm{H}$ dans la $i$-ème molécule gazeuse ; 


$$
K_{m}=\exp \left(-\Delta G_{m} / R T\right)
$$

$q_{i m}$ représente le coefficient de réaction de la $i$-ème espèce gazeuse dans la $m$-ième réaction.

La figure 2 montre les valeurs trouvées, calculées à partir des données thermochimiques de Yim [16], en fonction du rapport $(\mathrm{Cl} / \mathrm{H})$ pour deux températures.

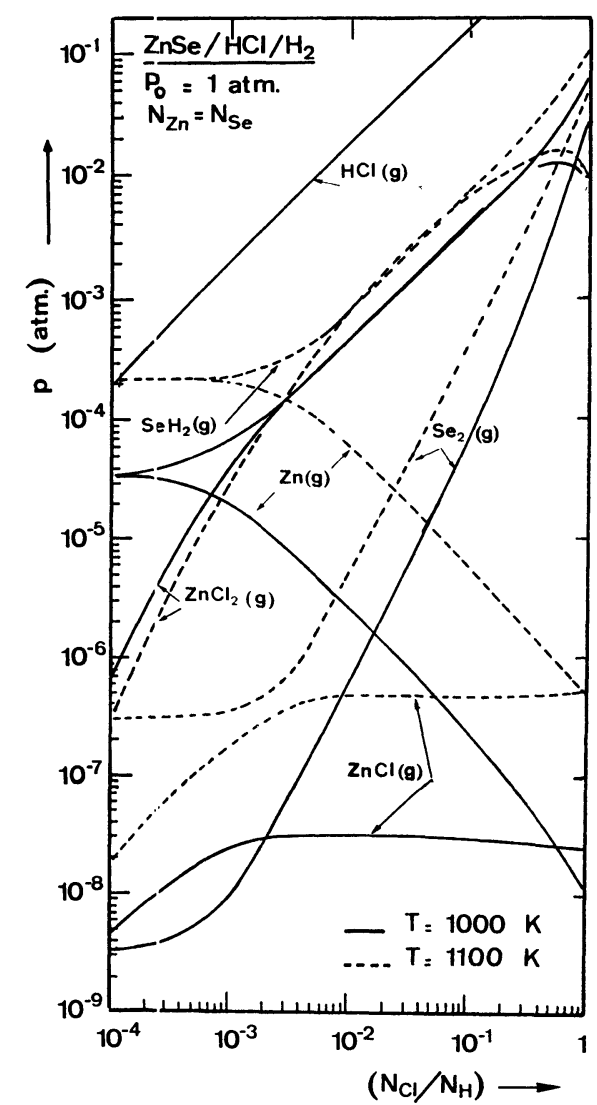

FIG. 2. - Pressions partielles d'équilibre, calculées à deux températures de source pour $10^{-4} \leqslant \mathrm{Cl} / \mathrm{H} \leqslant 1$.

[System $\mathrm{ZnSe}-\mathrm{HCl}-\mathrm{H}_{2}$ : partial pressures of equilibrium as a function of $(\mathrm{Cl} / \mathrm{H})$ for $T_{1}=1000 \mathrm{~K}$ and $1100 \mathrm{~K}$.]

La pression qui sera prise en compte dans notre problème sera due à la somme des pressions partielles des espèces à base de zinc $\left(p_{\mathrm{Zn}_{\mathrm{n}}}+p_{\mathrm{ZnCl}_{2}}\right)$ ou des espèces à base de sélénium $\left(2 p_{\mathrm{Se}_{2}}+p_{\mathrm{SeH}_{2}}\right)$. Pour un rapport $\mathrm{Cl} / \mathrm{H}$ de l'ordre de $10^{-4}$ ou inférieur, les pressions partielles principales correspondent à celles du système plus simple, représenté par les équilibres (II) et (III) - Transport à l'hydrogène seul.

4.2 CoffFICIENTS DE DIFFUSION. - En accord avec la méthode de Lennard-Jones, les coefficients binaires de diffusion ont été évalués à partir de la relation

$$
\mathfrak{D}_{\mathrm{AB}}=0,0009291 \frac{T^{3 / 2}}{P \sigma_{\mathrm{AB}}^{2} \Omega_{\mathrm{AB}}}\left(\frac{1}{M_{\mathrm{A}}}+\frac{1}{M_{\mathrm{B}}}\right)^{1 / 2}
$$

dans laquelle $M_{\mathrm{A}}$ et $M_{\mathrm{B}}$ sont les masses molaires de A et de $B, P$ (en atm.) la pression totale du mélange gazeux; $\sigma_{\mathrm{AB}}$ est une quantité indépendante de $T$ et définie par $2 \sigma_{\mathrm{AB}}=\sigma_{\mathrm{A}}+\sigma_{\mathrm{B}}, \sigma_{\mathrm{A}}$ et $\sigma_{\mathrm{B}}$ étant les diamètres des espèces moléculaires $A$ et $B$.

$\Omega_{\mathrm{AB}}$ est l'intégrale d'interaction, fonction sans dimension de la température. Ces différentes grandeurs sont tabulées dans de nombreux ouvrages ([17] à [19]).

Nous avons donc calculé un certain nombre de coefficients binaires tels que $\mathfrak{D}_{\mathrm{H}_{2}-\mathrm{HCl}}, \mathfrak{D}_{\mathrm{SeH}_{2}-\mathrm{HCl}}$, $\mathfrak{D}_{\mathrm{Zn}-\mathrm{HCl}}$, mais l'intégration de l'équation principale nécessite la connaissance d'un coefficient global de diffusion tenant compte de la proportion en $\mathrm{HCl}(\mathrm{g})$ et de l'espèce principale issue de la réaction avec $\mathrm{ZnSe}(\mathrm{s})$. Hirschfelder a proposé ([18]) pour un mélange ternaire :

$D_{\mathrm{AB}}=\mathscr{D}_{\mathrm{AB}}\left(1+\frac{x_{\mathrm{c}} \frac{M_{\mathrm{C}}}{M_{\mathrm{B}}}\left(\mathscr{D}_{\mathrm{AC}}-\mathfrak{D}_{\mathrm{AB}}\right)}{x_{\mathrm{A}} \mathfrak{D}_{\mathrm{BC}}+x_{\mathrm{B}} \mathscr{D}_{\mathrm{AC}}+x_{\mathrm{C}} \mathscr{D}_{\mathrm{AB}}}\right)$

$x_{\mathbf{i}}$ correspondant à la proportion de l'espèce " $i$ " dans le mélange gazeux. Nous avons ainsi évalué nos différents coefficients et avons choisi dans les deux cas de transport étudiés $\left(\mathrm{H}_{2}\right.$ et $\left.\mathrm{H}_{2}+\mathrm{HCl}\right)$ celui de l'espèce principale à base de sélénium, à savoir $\mathfrak{D}_{\mathrm{SeH}_{2}-\mathrm{H}_{2}}$ ou $\left(D_{\mathrm{SeH}_{2}-\mathrm{H}_{2}}\right)_{\mathrm{HCl}}$; en effet, c'est l'espèce $\mathrm{SeH}_{2}(\mathrm{~g})$ qui diffuse le moins vite dans le mélange gazeux.

5. Comparaison avec l'expérience. - Sur la figure 3 ont été reportées les courbes théoriques du flux

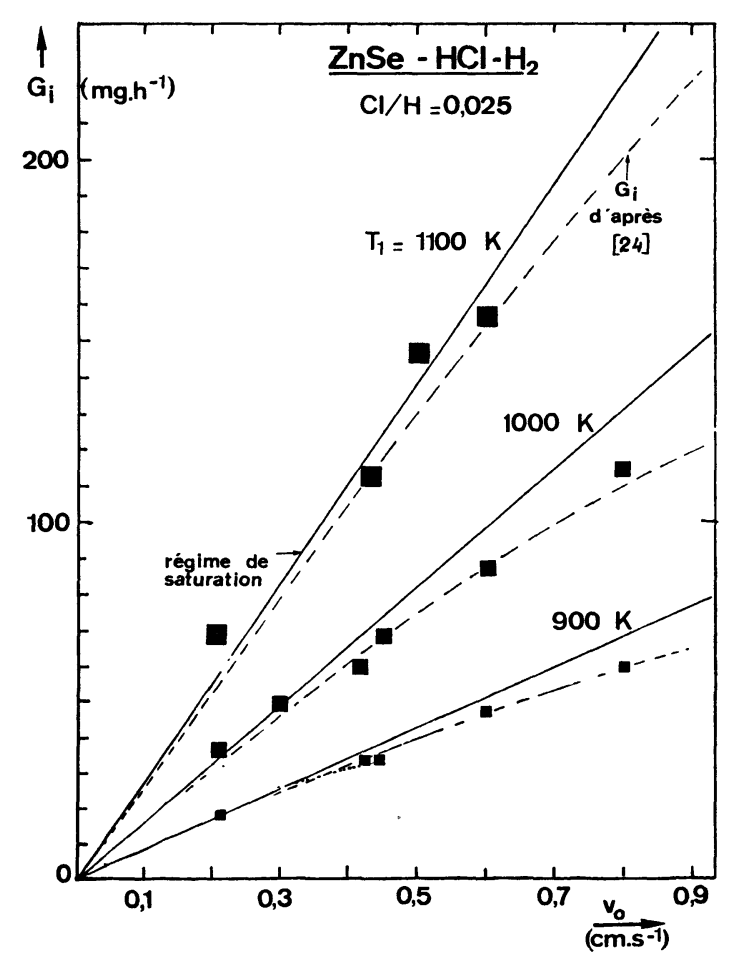

Fig. 3. - Système $\mathrm{ZnSe}-\mathrm{HCl}-\mathrm{H}_{2}$ : Comparaison des variations des bilans massiques expérimentaux en fonction de $v_{0}$ avec les variations calculées d'après l'expression (24).

[System ZnSe-HCl-H $\mathrm{H}_{2}$ : Theoretical (-) and experimental (- - -) values of the interfacial flux $G_{1}$ as a function of $v_{0}$ and $T_{1}$ for $(\mathrm{Cl} / \mathrm{H})=0,025(\mathrm{l}=4 \mathrm{~cm})$. 
d'interface $G_{i}$, calculé d'après l'expression (24), comparativement aux bilans massiques expérimentaux réalisés à la source, en fonction de la vitesse d'entraînement $v_{0}$ et de la température $T_{1}$, pour une valeur de $\mathrm{Cl} / \mathrm{H}$ fixée (ici 0,025).

Aux faibles vitesses, les points expérimentaux se distribuent sur la droite de saturation $(a d) v c_{1}$, où $(a d)$ a été remplacé par la section $S$ du tube $\left(7 \mathrm{~cm}^{2}\right)$, et vers laquelle tend $G_{i}$ lorsque $v_{0}$ tend vers 0 . Quand $v_{0}$ augmente, la sous-saturation de la phase vapeur augmente : cet état est bien décrit par la variation, tant expérimentale que théorique, de $G_{i}$.

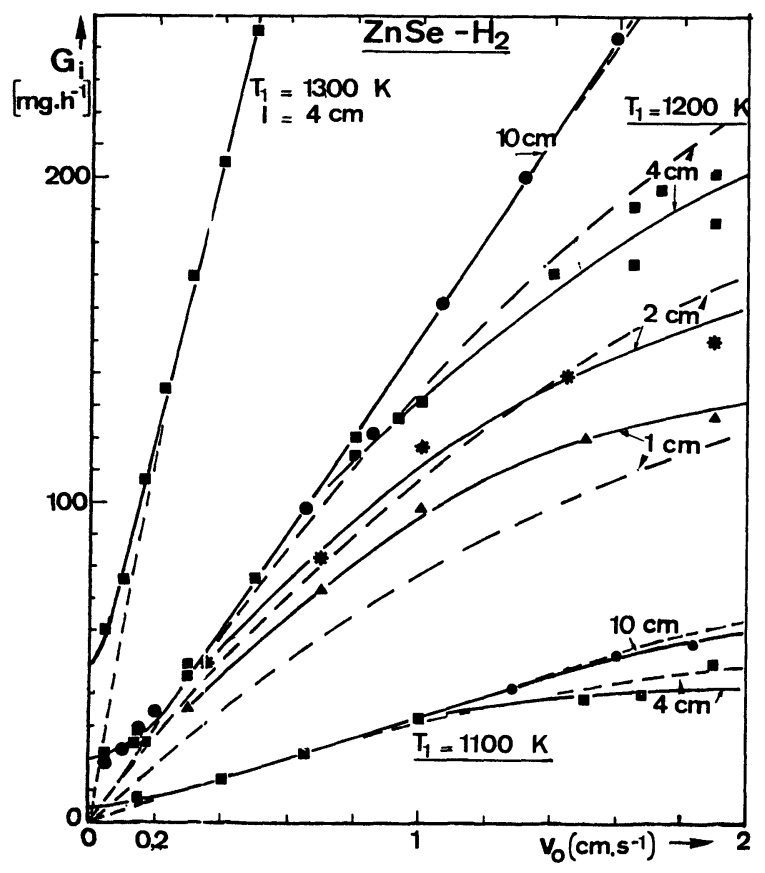

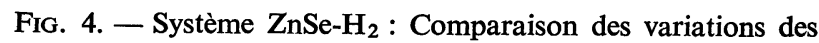
bilans massiques expérimentaux en fonction de $v_{0}$ avec les variations calculées d'après l'expression (24), pour différentes longueurs de source (de 4 à $10 \mathrm{~cm}$ ).

[System $\mathrm{ZnSe}: \mathrm{H}_{2}-(\mathrm{Cl} / \mathrm{H}=0)$ : Theoretical $(-)$ and experimental (- - ) values of the interfacial flux $G_{\mathrm{i}}$ as a function of $v_{0}$ and $T_{1}$, for different values of $l(1$ à $10 \mathrm{~cm})$.]
Nous avons mieux mis en évidence cette tendance pour le cas limite $\mathrm{Cl} / \mathrm{H}=0$ (Fig. 4) pour lequel nous avons, en plus, fait varier la longueur de source « $l$ ». Pour $l=10 \mathrm{~cm}$, le régime de saturation est couvert quel que soit $v_{0}$ (du moins, dans notre domaine d'étude). Pour des valeurs inférieures, le désaccord avec l'expérience n'excède pas $10 \%$, ce qui est remarquable si l'on se referre aux diverses hypothèses simplificatrices.

6. Discussion - Conclusion. - Ainsi, à partir d'expressions générales, adaptées à un problème de transfert bien précis, mais que l'on retrouve encore bien souvent dans l'élaboration de composés semiconducteurs, nous avons décrit d'une manière satisfaisante le transport de $\mathrm{ZnSe}$, depuis une zone source, en fonction de la température, de la vitesse d'entraînement, de la longueur de source et de la composition du gaz vecteur.

L'adoption d'un flux moyen nul à l'entrée de la zone source entraîne que l'expression établie, pour le flux de transfert, n'est valable qu'aux moyennes et grandes vitesses, c'est-à-dire, lorsque le flux de rétrodiffusion vers l'amont, en $x<0$, est négligeable ou nul. Cest effectivement le cas expérimentalement.

On peut cependant tenir compte des régimes non isothermes existant en réalité de part et d'autre de la source. Il suffit de calculer à partir d'une équation analogue à (2), les flux de rétrodiffusion $G_{2}^{\prime}$ et de simple diffusion $G_{2}$, dans les zones de transfert $2^{\prime \prime}$ et 2 ou sont supposées régner des températures moyennes $T_{2}^{\prime}$ et $T_{2}$. Nous avons présenté ces calculs dans un article déjà paru [20]. La sommation des trois flux $G_{i}, G_{2}^{\prime}$ et $G_{2}$ permet de rendre compte de toute la gamme de vitesse explorée expérimentalement.

Compte tenu des résultats obtenus, les hypothèses de départ semblent relativement justifiées et il semble même que ce modèle puisse être appliqué avec succès au transfert d'autres matériaux. C'est ce que nous nous employons actuellement à prouver.

\section{Bibliographie}

[1] Mandel, G., J. Phys. Chem. Solids 23 (1962) 587.

[2] Lever, R. F., J. Chem. Phys. 37 (1962) 1174.

[3] Arizumi, T., Nishinaga, T., Japan J. Appl. Phys. 4 (1965) 165.

[4] Carruthers, J. R., J. Cryst. Growth 2 (1968) 1.

[5] Secrest, B. G., Boyd, W. W., Shaw, D. W., J. Cryst. Growth 10 (1971) 251

[6] Klosse, K., Ullersma, P., J. Cryst. Growth 18 (1973) 167.

[7] FAKTOR, M. M., GARRET, I., Growth of Crystals from the Vapour (Chapman and Hall, London) 1975 Ch. V.

[8] Nölang, B. I., Richardson, M. W., J. Cryst. Growth 34 (1976) 198.

[9] Paorici, C., Pelosi, C., J. Cryst. Growth 35 (1976) 65.

[10] Fergusson, R. R., Gabor, T., J. Electrochem. Soc. 111 (1964) 585.

[11] PaO, Y. P., Phys. Fluids 14 (1971) 306.
[12] Tamir, A., TaItel, Y., Chem. Eng. Science 28 (1973) 1921.

[13] Chevrier, J., Galibert, G., Etienne. D. et Bougnot (G.), J. Cryst. Growth 28 (1975) 109.

[14] Carslaw, H. S., JAEGER, J. C., Conduction of Heat in Solids, Oxford 1959.

[15] Jost, W., Diffusion in Solids (Acad.-Press, N. Y.) 1960.

[16] YIM, W. M., J. Electrochem. Soc. 119 (1972) 381.

[17] BiRd, R. B., Steward, W. E., Lighfoot, E. H., Transport Phenomena (Wiley, N. Y.) 1959.

[18] Hirschfelder, J., Curtiss, C., BiRd, R. B., Molecular theory of gases and liquids (Wiley, N. Y.) 1954.

[19] Perry, H., Perry's Chemical Engineers' Handbook (McGawHill Cie) 1963.

[20] Chevrier, J., Etienne, D., Bougnot, G., Revue Phys. Appl. 9 (1974) 315. 